\title{
Proposta de utilização da pegada hídrica como indicador socioeconômico e ambiental na gestão dos recursos hídricos no estuário do rio Macaé
}

\author{
Édson Avelar Guimaraes ${ }^{1}$ \\ Universidade Federal do Rio de Janeiro - UFRJ \\ Elis Regina Nuffer Nunes ${ }^{2}$ \\ Universidade Federal do Rio de Janeiro - UFRJ/NUPEM \\ Vinicius Vanderley Miguel da Silva ${ }^{3}$ \\ Prefeitura Municipal de Macaé/RJ \\ Angelica Oliveira Carvalho 4 \\ Prefeitura Municipal de Macaé/RJ \\ Giseli Gomes dos Santos 5 \\ Instituto Federal Fluminense - IFF
}

\begin{abstract}
RESUMO
A água é recurso essencial à vida e buscar ferramentas que proporcionem uma gestão mais eficiente desse bem natural associando a questões socioeconômicas, sobretudo em assentamentos sem saneamento básico, torna-se fundamental à luz do preconizado pelos Objetivos de Desenvolvimento Sustentável (ODS) 6 e 11 da Agenda 2030 da ONU: assegurar a disponibilidade, a gestão sustentável da água e do saneamento básico; e garantir que as cidades e assentamentos humanos devam ser inclusivos, seguros, resilientes e sustentáveis, respectivamente. Objetiva-se testar a Pegada Hídrica $(\mathrm{PH})$, originalmente desenvolvida para medir o consumo direto e indireto de água, associada a dados socioeconômicos e ambientais em um assentamento precário no estuário do rio Macaé, no município de Macaé, RJ, como indicador capaz de auxiliar na gestão dos recursos hídricos. A metodologia consistiu em comparar a PH dos itens que compõem a cesta básica, dada a inexistência de abastecimento domiciliar de água potável e ausência de saneamento na localidade, com a quantidade de água disponibilizada àquela população por meio de caixas d'água comunitárias. O resultado indica uma PH do consumo indireto de água, presente nos itens mínimos de sobrevivência, muito superior à quantidade de água disponibilizada àquela população, contrariando as metas estabelecidas nos ODS 6 e 11. Conclui-se que a PH, além de medir o consumo de água, também pode indicar a sua carência e ser adaptada como indicador capaz de avaliar dados ambientais associados a condições socioeconômicas da população, propiciando uma gestão mais eficiente e equitativa dos recursos hídricos.
\end{abstract}

Palavras-chave: Água, Saneamento Básico, Objetivos de Desenvolvimento Sustentável

\begin{abstract}
Water is an essential resource for life and seeking tools that provide a more efficient management of this natural asset, associating it with socioeconomic issues, especially in settlements without basic sanitation, becomes essential in the light of the recommendations of the Sustainable Development Goals (SGD) 6 and 11 the UN 2030 Agenda: ensuring the availability, sustainable management of water and basic sanitation; and ensuring that cities and human settlements must be inclusive, safe, resilient and sustainable, respectively. The objective is to test the Water Footprint (PH),
\end{abstract}

\footnotetext{
1 Engenheiro Agrimensor da Prefeitura Municipal de Macaé. Doutorando em Ciências Ambientais e Conservação pela Universidade Federal do Rio de Janeiro - UFRJ. ${ }^{2}$ Prefeitura Municipal de Macaé. Mestranda em Ambiente, Sociedade e Desenvolvimento do Instituto de Biodiversidade e Desenvolvimento (Nupem/UFRJ-Macaé). ${ }^{3}$ Graduação em Engenharia Civil pela Universidade Estácio de Sá (2011). Engenheiro Civil da Prefeitura Municipal de Macaé. ${ }^{4}$ Doutora em Politica Social pela Universidade Federal Fluminense (2016); Mestre em Serviço Social (2009) e graduação em Serviço Social (2000). Atualmente é assistente social da Prefeitura Municipal de Macaé

${ }^{5}$ Arquiteta e Urbanista e mestranda em Arquitetura, Urbanismo e Tecnologias pelo Instituto Federal de Educação, Ciência e Tecnologia Fluminense - IFF.
} 
originally developed to measure direct and indirect water consumption, associated with socioeconomic and environmental data in a precarious settlement in the Macaé River estuary, in the municipality of Macaé, RJ, as an indicator capable of assist in the management of water resources. The methodology consisted of comparing the PH of the items that make up the basic basket, given the inexistence of household drinking water supply and lack of sanitation in the locality, with the amount of water available to that population through community water tanks. The result indicates a $\mathrm{PH}$ of indirect water consumption, present in the minimum items of survival, much higher than the amount of water available to that population, contrary to the goals established in SGD 6 and 11 . It is concluded that PH, in addition to measuring consumption of water can also indicate its shortage and be adapted as an indicator capable of assessing environmental data associated with the socioeconomic conditions of the population, providing a more efficient and equitable management of water resources.

Keywords: Water, Basic Sanitation, Sustainable Development Goals

\section{Introdução}

A água é um recurso essencial à vida e vem adquirindo, nas últimas décadas, importância socioeconômica, política e cultural cada vez maior devido à sua irregular distribuição espacial no planeta.A sua escassez, em algumas partes do globo, já é considerada uma ameaça global, conforme destacado na $10^{a}$ edição do Fórum Econômico Mundial ocorrido em Genebra, em 2015 (Brauman, Richter, Postel, Malsy, M \& Florke, 2016).

O grande crescimento populacional dos últimos anos promoveu um significativo impacto econômico e ambiental na exploração de recursos naturais, sobretudo nos recursos hídricos, para a produção de bens e produtos para atender uma demanda crescente de consumo da sociedade (Hoekstra, 2017).

A grande demanda por moradia, sobretudo no meio urbano, tem levado à ocupação irregular e desordenada do solo e, na maioria das vezes, esse processo promove a segregação socioespacial, gerando favelização com ocupação de áreas ambientalmente frágeis, degradando os recursos hídricos, o solo, a saúde dos moradores e desencadeando conflitos socioambientais ao contrapor interesses dos habitantes locais com a preservação e recuperação de recursos coletivos como mananciais e corpos d'água (Braga, 2001) e (Silva Neto, 2007).

Esse processo conduz a uma crescente demanda por água, à sua escassez e à sua degradação e tem colocado o debate sobre a gestão dos recursos hídricos presente nas discussões em todo o mundo, com o intuito de avaliar e de mensurar os impactos resultantes do manejo e do uso desses recursos (Haddeland et al., 2014); (Ercin \& Hoekstra, 2014) e (Ribeiro \& Dantas, 2017).

A avaliação da qualidade do meio ambiente, dada a situação de degradação e exploração crescente dos recursos naturais, tem sido conduzida à luz de indicadores de sustentabilidade que avaliam e integram aspectos sociais, econômicos e ambientais e, dentre esses indicadores, destacam-se aqueles relacionados à sustentabilidade dos recursos hídricos (Kemerich, Ritter \& Borba, 2014).

As comunidades e o ambiente necessitam de uma gestão eficiente dos recursos naturais, principalmente das águas, para que a vida silvestre e os solos sejam preservados e o desenvolvimento local seja atingido de forma sustentável (Freitas dos Santos, 2016).

\section{Gestão dos recursos hídricos e desenvolvimento sustentável}

Cuidado especial tem sido dispensado à gestão dos recursos hídricos quando se observa a distribuição desse bem no planeta: a água que se encontra livre constitui recursos hídricos e, aproximadamente, $97,2 \%$ dessa água é salgada; $2,8 \%$ correspondem a água doce; dos quais, na forma de glaciares, têm-se 2,15\%; em aquíferos subterrâneos, 0,63\%; em vapor d'água, 0,005\%; e, presente em rios e lagos, 0,01\% (Von Sperling, 2006).

A gestão sustentável da água deve considerar um novo modelo de desenvolvimento incorporado ao conceito de meio ambiente e à ideia do homem inserido no mesmo, além do próprio meio físico. É necessário entender que os meios físicos e socioeconômicos são fontes de recursos que dão suporte às atividades humanas e, ao mesmo tempo, são por elas impactados (Leal, 1998).

Sua relevância cresce devido aos bilhões de seres humanos excluídos do acesso à água em suas casas e da coleta e tratamento de esgotos ou quando se analisam inúmeros casos de 
doenças de veiculação hídrica e mortes por desidratação. Seu contorno final dá-se pelo desafio de atender às crescentes demandas de água da população humana, dos demais seres vivos que coabitam o planeta e, ao mesmo tempo, de preservar os ecossistemas naturais (Maranhão, 2007).

O tema tem ganhado importância quando se consideram danos enfrentados pelas populações ribeirinhas e pelo fato de muitas cidades brasileiras serem localizadas às margens de rios, lagoas e estuários, cujas águas têm sua qualidade deteriorada ou encontram-se sob permanente ameaça pela atividade humana (Guimarães, 2019).

Tal preocupação está caracterizada na Agenda 2030 da Organização das Nações Unidas (ONU), em seu plano de metas para um "desenvolvimento sustentável" composto por dezessete Objetivos de Desenvolvimento Sustentável (ODS), baseados no equilíbrio entre as três dimensões do desenvolvimento sustentável: a econômica, a social e a ambiental (ONU, 2016).

O ODS 6 deixa claro a necessidade de assegurar a disponibilidade e a gestão sustentável da água e do saneamento básico para todos. Por sua vez, o ODS 11 vem ao encontro do ODS 6 ao destacar que as cidades e os assentamentos humanos devem ser inclusivos, seguros, resilientes e sustentáveis (Carvalho \& Barcellos, 2015).

Os recursos hídricos, assim como os serviços a ele relacionados, sustentam os esforços de erradicação da pobreza, de crescimento econômico e sustentabilidade ambiental. O ODS-6 importa para todos os aspectos da dignidade humana: da segurança alimentar e energética à saúde humana e ambiental (Programa das Nações Unidas para o Desenvolvimento [PNUD] \& Instituto de Pesquisa Econômica Aplicada [IPEA], 2018).

Nesse contexto, a gestão dos recursos hídricos, definida como o processo de planejamento, coordenação e controle das atividades ligadas ao uso racional desses recursos, requer a utilização de indicadores adequados que auxiliem nas tomadas de decisão que são seu ponto essencial (Maranhão, 2007).

\section{Pegada hídrica}

Um indicador que vem sendo muito utilizado na gestão de recursos hídricos é a Pegada Hídrica, termo usado, inicialmente, a partir da abordagem da "água virtual", conceito criado em 1993 pelo cientista inglês John Anthony Allan. A “água virtual” avalia a quantidade de água consumida na produção de um bem, produto ou serviço, embutida no produto, não apenas no sentido visível, físico, mas, também, no sentido "virtual”, considerando a água utilizada nos processos produtivos, ou seja, é uma medida indireta dos recursos hídricos consumidos por um bem. Esse conceito explica a quantidade de água empregada para gerar um produto em um determinado local, porém, destinado para outra localidade, criando assim um fluxo virtual entre lugares (Figueiredo, 2016).

De acordo com (Hoekstra et al., 2011), a pegada hídrica oferece suporte à gestão dos recursos hídricos pelo seu vasto escopo de atividades que se estruturam e até se confundem com gestão propriamente dita, em quatro fases distintas:

(1) Definição de objetivos e escopo;

(2) Contabilização da pegada hídrica;

(3) Avaliação da sustentabilidade da pegada hídrica;

(4) Formulação de respostas à pegada hídrica.

A definição de pegada hídrica estabelece três tipos distintos: (i) a pegada hídrica azul, que se refere ao consumo das águas superficiais e subterrâneas de um produto ao longo de sua cadeia produtiva; (ii) a pegada hídrica verde, que está relacionada ao consumo de água de chuva, desde que esta não escoe e; (iii) a pegada hídrica cinza, que se refere à poluição e é definida como o volume de água doce necessário para assimilar a carga poluente, a partir de concentrações naturais e de padrões de qualidade das águas existentes. A soma das três pegadas hídricas representa a pegada hídrica total do produto, comunidade, indivíduo, bacia, consumidor, dentre outros (Hoekstra et al., 2011).

A pegada hídrica, portanto, oferece uma perspectiva adequada e ampla a respeito de 
como a população se relaciona com o uso da água doce. Trata-se de uma medida volumétrica de consumo e poluição da água. O impacto ambiental local de certa quantidade de consumo e poluição desses recursos depende da vulnerabilidade do sistema hídrico local e do número de consumidores e poluidores que fazem uso do mesmo sistema (Hoekstra et al., 2011).

Objetiva-se testar a Pegada Hídrica $(\mathrm{PH})$, originalmente desenvolvida para medir o consumo direto e indireto de água, associada a dados socioeconômicos e ambientais em um assentamento precário no estuário do rio Macaé, no município de Macaé, RJ, como indicador capaz de auxiliar na gestão dos recursos hídricos.

O emprego da pegada hídrica fornece informações espacial e temporalmente específicas sobre como a água é apropriada para os vários propósitos humanos. Dessa forma espera-se que os resultados da pegada hídrica forneçam subsídios para uma melhor gestão do uso e alocação equitativos e sustentáveis dos recursos hídricos, além de formar uma boa base para a avaliação dos impactos ambientais, sociais e econômicos.

\section{Metodologia}

\section{Área de Estudo}

Contida na Zona Especial de Interesse Social - ZEIS Ilha Colônia Leocádia, a área de estudo, popularmente conhecida como Rio Novo, é parte de uma ilha artificial originada de obras de retificação do rio Macaé pelo extinto Departamento Nacional de Obras e Saneamento (DNOS), na década de 1960, localizada no estuário do rio Macaé (Macaé, 2010).

Trata-se de uma ocupação irregular, composta por assentamentos precários e com características rurais, com baixa taxa de ocupação, que apresentam atividades rurais de subsistência. Embora a comunidade esteja numa área de proteção ambiental, a sua ocupação ocorreu a partir de um programa de assentamento rural do Instituto Nacional de Colonização e Reforma Agrária - INCRA. A comunidade Rio Novo desenvolveu-se ao longo de faixas marginais de proteção do Rio Macaé, numa área caracterizada pelo ecossistema de mangue, no estuário do Rio Macaé.

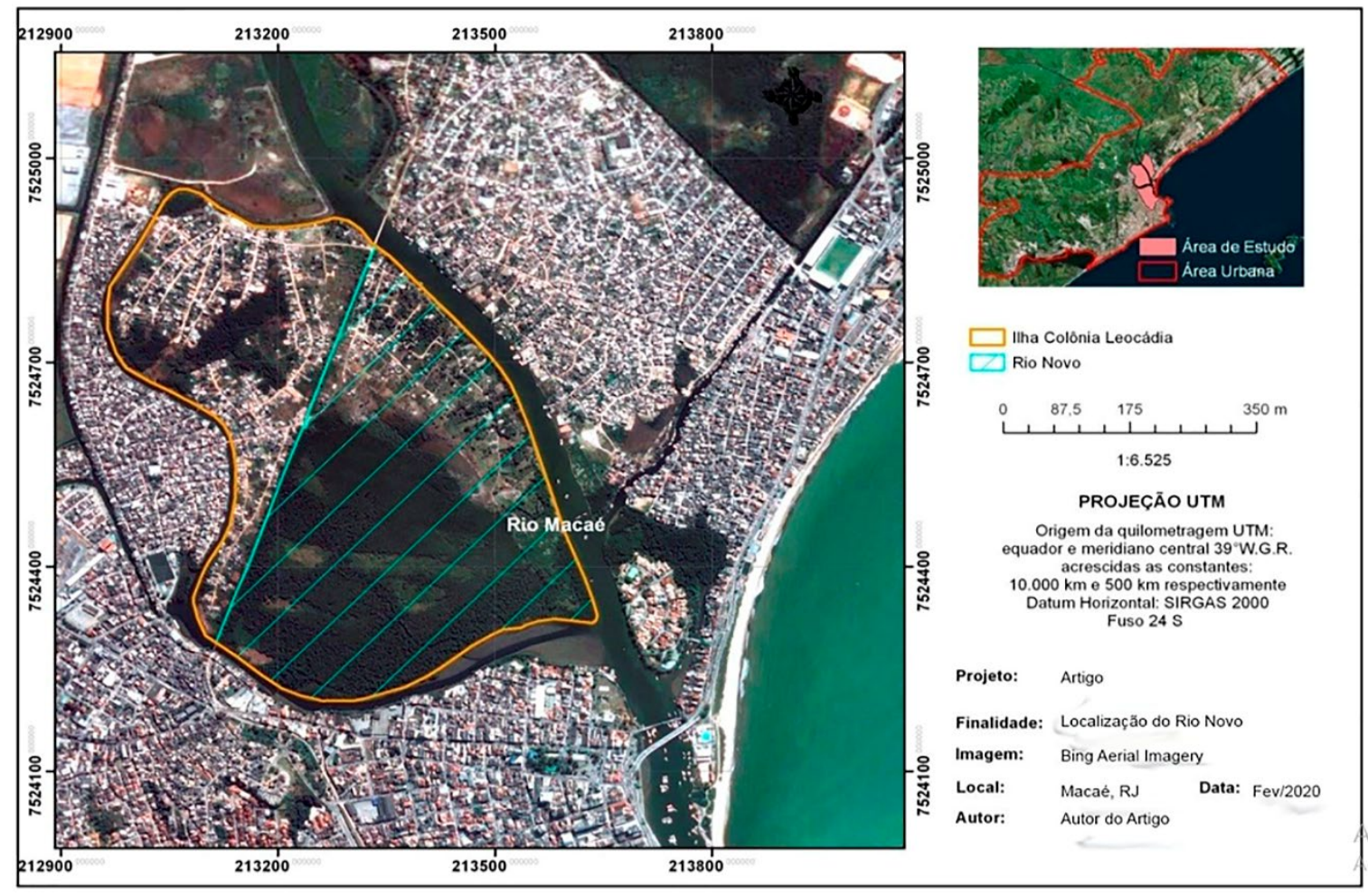

Figura 2.1: Comunidade Rio Novo no estuário do Rio Macaé ( Elaborado pelos autores). 
A localidade é desprovida de transporte e equipamentos públicos, sem pavimentação de vias, não apresenta sistema de abastecimento de água potável, não possui rede de drenagem,a coleta de lixo é precária e o esgotamento sanitário é lançado diretamente no solo e/ou no Rio Macaé.

\section{Estimativa da pegada hídrica azul para a comunidade Rio Novo}

O número de domicílios na comunidade é de 227 unidades habitacionais, com uma taxa média de ocupação de quatro pessoas por residência, totalizando uma população estimada de 908 habitantes (Macaé, 2016). O precário abastecimento de água existente dá-se por meio da disponibilização de cinco caixas d'água de 5.000 litros e uma de 10.000 litros abastecidas três vezes por semana, embora relatos de moradores indiquem uma periodicidade de abastecimento inferior.

Tabela 2.1 - Volume de água disponibilizado à população da localidade.

\begin{tabular}{|c|c|c|c|}
\hline \multicolumn{2}{|c|}{ POPULAÇÃO } & ÁGUA & ÁGUA \\
\hline Domicílios & Pessoas & Volume (m³/ano/domicílio) & $\begin{array}{l}\text { Volume } \\
\text { (m³/mês/localidade) }\end{array}$ \\
\hline 227 & 908 & 22,20 & $5.039,40$ \\
\hline
\end{tabular}

Fonte: Elaborado pelos autores.

\section{Water Footprint Network}

Segundo (Rocha, 2016), várias calculadoras têm sido utilizadas para facilitar a determinação dos valores da Pegada Hídrica direta e indireta, conforme observa-se em países como os Estados Unidos, Canadá e Portugal, contudo, a calculadora Water Footprint Network (Hoekstra et al., 2011) é a mais utilizada no mundo todo.

Com essa ferramenta é possível calcular a PH para indivíduos e comunidades mediante informações sobre país de origem, sexo, consumo de gêneros alimentícios diversos, uso doméstico da água, dentre outros dados (Rocha, 2016).

Em função das condições de vulnerabilidade socioeconômica e a ausência de dados sobre outras formas de consumo residencial e individual de água, será utilizada uma estimativa baseada nos produtos que compõem a cesta básica nacional.

De acordo com o Decreto Lei $\mathrm{n}^{\circ} 399$, de 1938, que regulamentou o Salário Mínimo Mensal, a cesta básica está atrelada ao salário mínimo que o trabalhador é capaz de adquirir e é composta dos seguintes itens:

Tabela 2.2 - Itens da Cesta Básica e a respectiva quantidade mensal

\begin{tabular}{|l|l}
\hline Itens da Cesta Básica & Quantidade Mensal / Pessoa \\
\hline Carne & $6,00 \mathrm{~kg}$ \\
\hline Leite & $15,00 \mathrm{l}=15,48 \mathrm{~kg}$ \\
\hline Feijão & $4,50 \mathrm{~kg}$ \\
\hline Arroz & $3,00 \mathrm{~kg}$ \\
\hline Farinha & $1,50 \mathrm{~kg}$ \\
\hline Batata & $6,00 \mathrm{~kg}$ \\
\hline Legumes & $9,00 \mathrm{~kg}$ \\
\hline Pão & $6,00 \mathrm{~kg}$ \\
\hline Café & $0,60 \mathrm{~kg}$ \\
\hline Frutas & $90,00 \mathrm{unid} .=7,74 \mathrm{~kg}$ \\
\hline Açúcar & $3,00 \mathrm{~kg}$ \\
\hline Banha/Óleo & $1,50 \mathrm{~kg}$ \\
\hline Manteiga & $0,90 \mathrm{~kg}$ \\
\hline
\end{tabular}

Fonte: Adaptado do Decreto Lei $\mathrm{n}^{\circ} 399$, de 1938 e DIEESE 2019

\section{Resultados e discussão}

Considerando os itens da cesta básica, conforme relação do Tabela 2.2, e utilizando-se 
a calculadora de pegada hídrica disponibilizada pela Water Footprint Network, pode-se chegar aos valores da pegada hídrica para a localidade, conforme a Tabela 3.1:

\begin{tabular}{|c|c|c|}
\hline Itens da Cesta Básica & $\begin{array}{l}\text { Pegada Hídrica/Domicílio } \\
\left(\mathrm{m}^{3 / a n o}\right)\end{array}$ & $\begin{array}{l}\text { Pegada Hídrica/Localidade } \\
\left(\mathrm{m}^{3} / \text { ano }\right)\end{array}$ \\
\hline Produtos Cereais & 881 & 199.987 \\
\hline Produtos Carne & 3026 & 686.902 \\
\hline Laticínios & 853 & 193.631 \\
\hline Vegetais & 115 & 26.105 \\
\hline Frutas & 261 & 59.247 \\
\hline Raízes & 119 & 27.013 \\
\hline Café e Açúcar & 658 & 149.366 \\
\hline PEGADA HÍDRICA TOTAL & 5.913 & 1.342 .251 \\
\hline
\end{tabular}

Fonte: Elaborada pelos autores.

Dessa forma, pode-se constatar que a Pegada Hídrica, por morador da localidade, é de aproximadamente $1.478 \mathrm{~m}^{3} / \mathrm{ano}$.

Esse valor adquire melhor compreensão, em ordem de grandeza, procedendo-se a comparações entre lugares geográficos e socioeconomicamente distintos, conforme o Tabela 3.2. Importante destacar que, normalmente, a $\mathrm{PH}$ dos países é determinada levando-se em consideração diversos fatores dentre os quais os mais relevantes são: volume de consumo em relação ao Produto Interno Bruto, padrão de consumo local, condições climáticas e práticas agrícolas (Giacomin \& Ohnuma, 2012).

Tabela 3.2 - Comparação da Pegada Hídrica do Rio Novo com outros lugares

\begin{tabular}{llll}
\hline Local & $\begin{array}{l}\text { Pegada } \\
\left(\mathbf{m}^{3} / \mathbf{a n o}\right)\end{array}$ & Hídrica & per capita \\
\hline Estados Unidos & 2842 & \\
\hline Brasil & 2027 \\
\hline Rio Novo & 1478 \\
\hline Congo & 552 & \\
\hline
\end{tabular}

Fonte: Adaptado de (Mekonnen \& Hoekstra, 2011) e (Roppa, 2006)

Generalizando, podem-se constatar valores maiores de pegada hídrica entre países economicamente mais desenvolvidos em relação aos países em desenvolvimento ou subindustrializados. Importante destacar que essa regra é geral, posto que a escassez de água tende a elevar o valor da pegada, ao passo que um consumo racional tende a diminuir o seu valor.

O valor encontrado para a localidade de estudo tende a se aproximar da média nacional, em parte, devido às condições semelhantes de desenvolvimento econômico e social da realidade brasileira. Nesse caso, foi considerada apenas a parcela relacionada ao uso indireto do recurso tendo como base uma dieta alimentar baseada nos produtos que compõem a cesta básica brasileira, razão pela qual pode-se esperar que, ao se acrescentar as parcelas relativas às outras formas de pegada hídrica, esse valor aumente, aproximando-se mais da média nacional.

Tão importante quanto as comparações explicitadas é relacionar a pegada hídrica local com a disponibilidade de água que atende a população daquela localidade. Conforme citado, o volume de água disponibilizado aos moradores é de aproximadamente 5,55 m3/ano por habitante, enquanto a respectiva pegada hídrica, relativa a alimentos, é de $1.478 \mathrm{~m} 3 / \mathrm{ano}$, ou seja, a água disponibilizada à população está na ordem de grandeza 268 vezes inferior ao que é utilizado no consumo mínimo da cesta básica.

Tabela 3.3- Comparação da Pegada Hídrica do Rio Novo com outros lugares

\begin{tabular}{|l|c|c|c|c}
\hline Pegada Hídrica Individual & EUA & BRASIL & MUNDO & Rio Novo \\
\hline Doméstico & 111 & 56 & 65 & 5,55 \\
\hline Alimentação & 2398 & 1926 & 1267 & 1478 \\
\hline $\begin{array}{l}\text { PH Alimentação/PH } \\
\text { Doméstico }\end{array}$ & 21 & 34 & 19 & 268 \\
\hline
\end{tabular}


Conforme a Tabela 3.3, pode-se perceber que a relação entre as pegadas hídricas da alimentação e do uso doméstico, para a localidade em estudo, destoa consideravelmente dos valores observados em países economicamente desenvolvidos, da realidade socioeconômica brasileira e da média mundial.

Importante destacar que a demanda por água daquela população é ainda superior aos valores apresentados, uma vez que o consumo relativo a outros usos, como higiene, limpeza, irrigação, dessedentação de animais, lazer, consumo direto e esgotamento sanitário, não foram contabilizados no presente estudo.

Para mitigar a situação de escassez de água, os moradores buscam pelo recurso em fontes alternativas de acordo com sua disponibilidade econômica. Alguns poucos podem pagar pelo abastecimento de caixas d'água por meio de caminhões-pipa e a grande maioria utiliza água salobra e, possivelmente, contaminada de poços freáticos ou captadas diretamente no rio Macaé.

\section{Conclusão}

Tendo em vista as três dimensões do desenvolvimento sustentável - a econômica, a social e a ambiental - e, à luz do preconizado pelos ODS 6 e 11, fica clara a necessidade de pensar em ações de gestão, sobretudo do poder público, que assegurem às pessoas que habitam aquela localidade o acesso digno à água potável e ao saneamento básico, considerando que os assentamentos humanos devem, minimamente, serem inclusivos, seguros, resilientes e sustentáveis.

Conforme Guimarães (2019), a situação de extrema vulnerabilidade hídrica, ambiental e socioeconômica dos moradores daquela localidade já apontava para uma condição de grave Pobreza Hídrica.

Tendo em vista a quarta fase proposta por Hoekstra et al. (2011), relativa à Formulação de respostas à pegada hídrica, em que a pegada hídrica deve dar suporte à gestão dos recursos hídricos, este trabalho sugere, embora mais estudos devam ser desenvolvidos, que a Pegada Hídrica pode ser empregada como indicador capaz de avaliar as condições de vulnerabilidade hídrica das pessoas e do respectivo ambiente.

Dessa forma, uma ferramenta originalmente desenvolvida, pensando-se em quantificar o tamanho do consumo de água, pode ser empregada visando à eficiência na gestão do recurso hídrico colaborando com os esforços em busca da erradicação da pobreza, do desenvolvimento socioeconômico e da sustentabilidade ambiental dos lugares e das pessoas.

\section{Referências}

Braga, T. (2001). Principais limitações à internalização da dimensão ambiental nas práticas urbanas. Rio de Janeiro. Anais do IX Encontro Nacional da ANPUR, p. 1322-1333.

Brasil. Decreto Lei n ${ }^{\circ}$ 399. (1938). Aprova o regulamento de execução da lei n. 185, de 14 de janeiro de 1936, que institui como Comissões de Salário Mínimo. Diário Oficial da União.

Brauman, K.A., Richter, B.D., Postel, S., Malsy, M. and Flörke, M. (2016). Water depletion: An improved metric for incorporating seasonal and dry-year water scarcity into water risk assessments. DOI: http://doi.org/10.12952/journal.elementa.000083

Carvalho, P. G. M., Barcellos, F. C. (2015). Os Objetivos de Desenvolvimento do Milênio: uma avaliação crítica. Rio de Janeiro: IBGE, Diretoria de Pesquisas, $52 \mathrm{p}$.

Departamento Intersindical de Estatística e Estudos Socioecômicos (2020). Metodologia da Cesta Básica de Alimentos. Disponível: https://www.dieese.org.br/metodologia/metodologiaCestaBasica/?page=1

Ercin, A. E., Hoekstra, A. Y. (2014). Water footprint scenarios for 2050: A global analysis. Environ. Int. 64, 71-82.

Figueiredo, M. C., Silva, E. H. B. C., Silva, S. M. (2015). Estimativa da Pegada Hídrica Cinza da Água Tratada em Feira de Santana, BA.

Fórum Econômico Mundial (2015). Global Risks. 10a Edição. Genebra: Fórum Econômico Mundial.

Freitas dos Santos, R. S.; Ferreira, M. I. P. (2016). Índice de Pobreza Hídrica e sua adaptação às condições da comunidade de Gargaú, São Francisco do Itabapoana/RJ. Boletim do Observatório Ambiental Alberto Ribeiro Lamego, v. 10, p. 191206. 
Giacomin, G. S.; Ohnuma, A. A. (2012). Análise de Resultados de Pegada Hídrica por Países e Produtos Específicos. Revista Eletrônica em Gestão, Educação e Tecnologia Ambiental, v. 8, p. 1562-1572.

Guimarães, E. A. ( 2019). Objetivos de Desenvolvimento Sustentável e Pobreza Hídrica: Estudo Comparativo de Comunidades Estuarinas do Município de Macaé/RJ. Macaé. (Dissertação de Mestrado). Instituto Federal Fluminense, Macaé, RJ.

Haddeland, I., Heinke, I., Biemans, H., Eisner, S., Flörke, M., Hanasaki, N., Konzmann, M., Ludwig, F., Masaki, Y., Schewe, J., Stacke, T., Tessler, Z. D., Wada, Y \& Wisser, D. (2014). Global water resources affected by human interventions and climate change. PNSA National Academy of Sciences, Alemanha.

Hoekstra, A. Y.; Chapagain, A. K.; Aldaya, M. M.; Mekonnen, M. M. (2011). The water footprint assessment manual: setting the global standard. [S.1.]: Earthscan. 224p.

Hoekstra, A.Y. Water Footprint Assessment: Evolvement of a New Research Field. Water Resour Manage 31, 3061-3081 (2017). https://doi.org/10.1007/s11269-017-1618-5.

Instituto de Pesquisa Econômica Aplicada (2018). SPI - Secretaria de Planejamento e Investimentos Estratégicos. Objetivos de Desenvolvimento Sustentável: Relatório Nacional de Acompanhamento. Brasília: 2018.

Kemerich, P. D. C., Ritter, L. G., BORBA, W. F. (2014). Indicadores de sustentabilidade ambiental: métodos e aplicações. Revista Monografias Ambientais- REMOA, v.13, n.5, ed. especial, p. 3723-3736.

Leal, M. S. (1998). Gestão Ambiental dos Recursos Hídricos: Princípios e Aplicações. CPRM. Rio de Janeiro.

Macaé. Prefeitura Municipal. Secretaria Municipal de Habitação (2010). Plano Local de Habitação de Interesse Social. Macaé.

Macaé. Prefeitura Municipal. Secretaria Municipal de Habitação (2016). Plano de Urbanização da Ilha Colônia Leocádia. Macaé.

Maranhão, N. (2007). Sistema de Indicadores para Planejamento e Gestão dos Recursos Hídricos de Bacias Hidrográficas. Rio de Janeiro: UFRJ.

Mekonnen, M. M., Hoekstra, A.Y. (2011) National water footprint accounts: the green, blue and grey water footprint of production and consumption. Value of Water Research Report Series, No. 50, UNESCO-IHE, Volume 1.

Organização das Nações Unidas (2016). Transformando nosso mundo: a Agenda 2030 para o desenvolvimento sustentável. Nova Iorque: Centro de Informação das Nações Unidas. 42 p.

Pesquisa Nacional por Amostra de Domicílios (2015). Síntese de Indicadores. Rio de Janeiro: IBGE.

Programa das Nações Unidas para o Desenvolvimento (2018). Plataforma Agenda 2030.

Ribeiro, G. A., Dantas Neto, J. (2017). Potencialidades e Normatização da Pegada Hídrica. In: Congresso Nacional de Meio Ambiente, 2017, Poço de Caldas. Anais Congresso Nacional de Meio Ambiente de Poços de Caldas. v. 09.

Rocha, C. M. R. de S. (2014). Calculadora da pegada hídrica residencial. (Dissertação de Mestrado). Universidade Federal de Campina Grande, Paraíba, Brasil.

Roppa, L. (2006) Perspectivas da produção mundial de carnes, 2006 a 2030. Revista Pork World, n.34, p.16-27.

Silva Neto R., Dias, R. S. ; Barral Neto, J. F. ; Rangel, L. C. (2007). Desafios para o desenvolvimento sustentável no município de Macaé - RJ. Perspectivas Online. Campos dos Goitacazes, v. 5, p. 60-73.

Von Sperling, E. (2006). Afinal, quanta água temos no planeta? Revista Brasileira de Recursos Hídricos (RBRH), v. 11, n.4. Out/Dezembro. p. 189-199.

Water Footprint Network (2020). Calculadora da Pegada Hídrica. Disponível em https://waterfootprint.org/en/resources/interactive-tools/personal-water-footprint-calculator/ 